\title{
Determinants and protective associations of the lupus low disease activity state in a prospective Chinese cohort
}

\author{
Yanjie Hao ${ }^{1,2}$ - Shereen Oon ${ }^{2,3} \cdot{\text { Lanlan } \mathrm{Ji}^{1} \cdot \text { Dai Gao }}^{1} \cdot$ Yong Fan ${ }^{1} \cdot$ Yan Geng $\cdot$ Xiaohui Zhang ${ }^{1} \cdot$ Guangtao $\mathrm{Li}^{1}$. \\ Eric F. Morand ${ }^{4} \cdot$ Mandana Nikpour $^{2,3} \cdot$ ZhuoLi Zhang $^{1}$
}

Received: 18 July 2021 / Revised: 20 September 2021 / Accepted: 23 September 2021 / Published online: 30 September 2021

(c) The Author(s) 2021

\begin{abstract}
Objective To investigate the frequency and determinants of achieving the lupus low disease activity state (LLDAS), and the effect of LLDAS attainment on disease flare and damage accrual in a prospective, single-center cohort of Chinese lupus patients.

Methods Baseline and follow-up data from consecutive patients at the Peking University First Hospital were collected from January 2017 to June 2020.

Results A total of 185 patients were enrolled, with median (range) disease duration at enrolment of 2.3 (0.8-7.7) years, and median follow-up of $2.2(1.0-2.9)$ years. By the end of the study, 139 (75.1\%) patients had achieved LLDAS at least once; 82 (44.3\%) patients achieved LLDAS for $\geq 50 \%$ of observations. Multivariable logistic regression analysis showed that 24-h urinary total protein (UTP; per $\mathrm{g})(\mathrm{OR}=0.447,95 \% \mathrm{CI}[0.207-0.968], p=0.041)$, serum creatinine (Scr; per $10 \mu \mathrm{mol} / \mathrm{L})$ $(\mathrm{OR}=0.72,95 \% \mathrm{CI}[0.52-0.99], p=0.040)$, and C3 level $($ per $100 \mathrm{mg} / \mathrm{L})(\mathrm{OR}=1.60,95 \% \mathrm{CI}[1.18-2.17], p=0.003)$ at recruitment had independent negative associations with achieving LLDAS for $\geq 50 \%$ of observations. Kaplan-Meier analyses showed a significant reduction in flare rate with increased proportion of time in LLDAS. Attainment of LLDAS in at least $50 \%$ of observations was an independent protective factor for damage accrual ( $\mathrm{OR}=0.19,95 \% \mathrm{CI}$ [0.04-0.99], $p=0.049$ ).

Conclusions In this prospective Chinese cohort, LLDAS was an attainable goal in clinical practice. Nephritis-related markers (UTP and Scr) and C3 level at recruitment negatively influenced achievement of LLDAS. LLDAS achievement was significantly protective from flare and damage accrual.

Key points

- Low disease activity status (LLDAS) is an achievable target during SLE treatment in China. Urine protein, serum creatinine, and C3 level at recruitment independently affect LLDAS achievement in this group of Chinese lupus patients.

- As a treatment target, LLDAS achievement has a highly protective effect for preventing flare and damage accrual, especially in case of achieving LLDAS for $\geq 50 \%$ of observations.

- The present results further highlight the practical significance of treat-to-target principle in SLE management (T2T/SLE) and the needs for promoting the application of T2T/SLE in clinical practice as well as exploring the concrete implement strategy.
\end{abstract}

Keywords Damage accrual $\cdot$ Disease flare $\cdot$ Low disease activity $\cdot$ Systemic lupus erythematosus $\cdot$ Treat-to-target

\section{Introduction}

Systemic lupus erythematosus (SLE) is a chronic multisystem autoimmune disease, where treatment is typically long term or even lifelong. With a paucity of advanced

ZhuoLi Zhang

zhuoli.zhang@126.com

Extended author information available on the last page of the article therapeutics, most patients are treated with systemic glucocorticoids in addition to immunosuppressants (IS). Despite these, irreversible organ damage and mortality remain unacceptably high. A recent study from the investigators' center reported that the standardized mortality rate (SMR) of SLE patients in China compared to general population was 3.2 [1]; a meta-analysis of 15 studies comprising 26,101 SLE patients of various ethnicities revealed 
a similar SMR of 2.7 [2]. Understanding of factors which lead to increased SLE morbidity is therefore paramount.

The principle of treat-to-target (T2T) has been successfully applied to many rheumatological and non-rheumatological diseases. For instance, T2T strategies have dramatically improved the prognosis of patients with rheumatoid arthritis. The concept of T2T in SLE was relatively more recently proposed [3], with several "remission" or "low disease activity" states proposed by an expert task force as treatment targets [4]. The definitions of remission in SLE (DORIS) criteria for remission and lupus low disease activity state (LLDAS) criteria from the Asia-Pacific Lupus Collaboration (APLC) for low disease activity were the most accepted and used definitions [5, 6]. The LLDAS has been both retrospectively and prospectively validated as being associated with reduced damage accrual in several studies [7-9], and found also to be more attainable than remission, whilst being no less protective [10]. With only one prospective validation study previously reported [6], the purpose of the present study was to investigate the frequency and determinants of achieving LLDAS, and the influence of LLDAS on disease flare and damage accrual in a single-center prospective longitudinal cohort of Chinese SLE patients.

\section{Materials and methods}

\section{Study population}

Baseline and follow-up data are prospectively collected from all consecutive patients treated at the Peking University First Hospital in Beijing. Ethics approval was obtained from the ethics committee of the Peking University First Hospital (Project Number: 2017[1284]), and written informed consent obtained from all participants. Subjects fulfilled either the 1997 American College of Rheumatology (ACR) updated classification criteria for SLE [11] or the 2012 Systemic Lupus International Collaborating Clinics (SLICC) classification criteria [12]. No specific treatment algorithm was predefined. Patients were usually followed every 3 months, with 6 months as the maximum allowable interval between consecutive visits. Data from adult SLE patients ( $\geq 18$ years) with at least one follow-up visit between January 2017 and June 2020 were analyzed in this study. Incident patients were defined as the patients who were recruited within 1 year of the onset of their SLE symptoms. Patients were considered lost to follow-up if they had no data recorded for 12 months, and were unable to be contacted after two attempts. The data for these patients collected prior to their loss to follow-up was included in the analysis.

\section{Data collection}

The demographics, disease duration at recruitment, follow-up duration, SLE-related manifestations, and organ involvement, as determined by the ACR classification criteria on an "ever present" basis, were collected. SLEDAI2K [13], PGA (scale 0-3) [14], and disease flare assessed using the SLE flare index (SFI) [15] were collected at each visit. Irreversible disease damage was captured using the Systemic Lupus International Collaborating Clinics Damage Index (SDI) [16] and health-related quality of life was captured using the short form (36) health survey (SF36) [17], both annually. Damage accrual was defined as an increase of $\geq 1$ in SDI. All data were recorded in a standardized electronic case report form as part of the APLC longitudinal cohort study [18]. Of note, data in the present study have not been previously included in APLC publications due to the recency of the Peking University Hospital joining this collaboration. Current use and doses of glucocorticoids, hydroxychloroquine (HCQ) and IS, and laboratory results including complete blood count, renal function, serum albumin (ALB), 24-h urine total protein (UTP), complement levels (C3 and C4), and anti-double stranded DNA (dsDNA) antibody titers at baseline and each follow-up visit were also collected.

\section{Definitions}

Cutaneous and mucosal involvement, leukopenia, thrombocytopenia, and serositis were defined using the 2012 SLICC criteria [12]. Lupus nephritis (LN) was defined as (i) proteinuria $>0.5$ grams per day or $>3+$, or (ii) cellular casts that may be red cell, hemoglobin, granular, tubular, or mixed, or (iii) biopsy-proven nephritis compatible with SLE $[12,19]$. Neuropsychiatric lupus (NPSLE) included a series of disorders ranging from diffuse central nervous system (CNS) disorders (acute confusional state, psychosis, anxiety and depression, and clinical to subclinical cognitive disorders with variable functional significance) to focal CNS syndromes (seizures, cerebrovascular diseases, chorea, myelopathy, transverse myelitis, demyelinating syndrome, aseptic meningitis, headaches) and peripheral nervous system disorders (polyneuropathies, mononeuropathies, autonomic disorders, plexopathy, acute inflammatory demyelination and polyradiculo-neuropathy) [20, 21]. The diagnosis of autoimmune hemolytic anemia (AIHA) was based on evidence of hemolysis including reticulocytosis, bilirubinemia, increased lactate dehydrogenase (LDH), and a positive direct antiglobulin test [22]. Pulmonary arterial hypertension (PAH) was defined as a mean pulmonary artery pressure $\geq 25 \mathrm{mmHg}$ and a 
pulmonary arterial wedge pressure $\leq 15 \mathrm{mmHg}$ by right heart catheterization, or a mean estimated pulmonary systolic pressure $\geq 50 \mathrm{mmHg}$ on echocardiography [23].

LLDAS was defined as an SLE disease activity index (SLEDAI)-2 $\mathrm{K}$ of $\leq 4$, no activity in any major organ system and no features of new disease activity, a physician global assessment (PGA, $0-3) \leq 1$, prednisone dose $\leq 7.5 \mathrm{mg} /$ day, and allowance for maintenance IS and anti-malarials [6].

\section{Data analysis}

Data are reported as mean (standard deviation (SD)) for normally distributed continuous variables, median (interquartile range (IQR)) for skewed continuous data, and percentages or proportions for categorical variables. The Student's $t$-test was used for comparisons of normally distributed continuous variables, Kruskal-Wallis and Mann-Whitney $U$ tests for comparisons of non-normally distributed continuous variables, and the chi-squared test for comparisons of categorical data. Univariate and multivariable logistic regression was used to identify factors associated with achieving LLDAS or damage accrual. Variables with a $p$ value $\leq 0.05$ in simple logistic regression analyses were included in stepwise multivariable regression analysis. Variables which relate directly to LLDAS assessment such as SLEDAI, PGA, and prednisone dose were not included in the logistic regression model for achieving LLDAS. Flare rate analysis during the follow-up was performed using the Kaplan-Meier method with comparisons performed using the log-rank test. The primary endpoint was disease flare or data censoring. The duration of follow-up was defined as the time from recruitment until the first flare or last follow-up.

All analyses were performed with STATA version 13.1 (StataCorp, College Station, TX, USA) for Windows and a $p$-value of $<0.05$ was considered statistically significant.

\section{Results}

\section{Subject characteristics}

Between January 2017 and June 2020, 200 SLE patients were recruited in the prospective cohort. After excluding 15 patients who did not return for follow-up after the first visit, 185 patients including 54 incident patients $(29.2 \%)$ with total 1203 visits were included in this study. The mean age at disease onset was $33.5 \pm 14.9$ years with a female predominance $(88.1 \%)$. The median (IQR) duration at recruitment was $2.3(0.8-7.7)$ years, median follow-up duration was 2.2 (1.0-2.9) years, and median number of visits was 7 (4-9). Among the 185 patients, 19 patients were lost to follow-up.

In terms of clinical characteristics, cutaneous and mucosal involvement was most common $(61.6 \%$ of the patients), followed by LN (55.7\%), leukopenia (44.3\%), arthritis $(39.5 \%)$, thrombocytopenia $(25.4 \%)$, serositis (15.3\%), AIHA $(9.7 \%)$, and NPSLE $(8.7 \%)$. Other less common disease manifestations included myositis (6.5\%), gastrointestinal tract involvement (6.0\%), and PAH (4.3\%). Renal biopsy was conducted in $62(33.5 \%)$ patients. The median (IQR) SLEDAI-2 K, PGA, and SDI at recruitment were $2(2-6), 1(1-2)$, and $0(0-1)$, respectively. One hundred and eighty-two patients $(98.4 \%)$ received glucocorticoid treatment, $171(92.4 \%)$ received HCQ, and $170(91.9 \%)$ received IS for at least 3 months. During follow-up, 111 (82.1\%) patients received IS for at least 6 months and 109 (73.2\%) for at least 12 months.

\section{Frequency of LLDAS attainment}

In the 185 patients, 58 (31.4\%) patients fulfilled LLDAS at recruitment, $81(43.8 \%)$ patients achieved LLDAS during follow-up, and 45 (24.3\%) never achieved LLDAS. Therefore, there were 139 (75.1\%) patients who achieved LLDAS at least once, including $29(15.7 \%)$ patients who were in LLDAS for $100 \%$ of observations, 53 (28.7\%) for 50 to $100 \%$ of observations, and 58 (31.4\%) in LLDAS for $<50 \%$ of observations (Fig. 1A). In a subgroup of 144 patients who completed at least 12 months of follow-up, the attainment of LLDAS during the follow-up were shown in Fig. 1B.

\section{Characteristics of patients achieving LLDAS attainment for $\geq 50 \%$ of observations}

The characteristics of patients who achieved LLDAS for $\geq 50 \%$ of observations were compared to those who did not (Table 1). Compared with subjects who achieved LLDAS for at least $50 \%$ of observations (referred to as the LLDAS $\geq 50 \%$ group), those who achieved LLDAS for less than $50 \%$ of observations (referred to as LLDAS $<50 \%$ group) had a higher UTP (390 [50-2300] vs 50 [20-150] mg, $p<0.001)$, lower albumin $(39.2 \pm 5.3$ vs $42.4 \pm 5.2 \mathrm{~g} / \mathrm{L}, p<0.001)$, higher Scr $(86.7 \pm 34.2$ vs $75.6 \pm 17.2, p=0.027)$, lower C3 $(714 \pm 209$ vs $857 \pm 200 \mathrm{mg} / \mathrm{L}, p<0.001)$, higher SLEDAI-2 K (4 [2-6] vs $2[0-2], p<0.001)$, higher PGA $(1[1,2]$ vs $0[0-1]$, $p<0.001)$, and higher prednisone dose $(11.9 \pm 3.9 \mathrm{vs}$ $5.4 \pm 2.6 \mathrm{mg}, p<0.001)$ at recruitment. The LLDAS $<50 \%$ group had a significantly higher proportion of patients who experienced a disease flare during the follow up period $(58.6 \%$ vs $27.5 \%, p<0.001)$ and were more likely to have damage accrual after 2 years of follow-up (48.8\% vs $21.4 \%, p=0.004)$ than the LLDAS $\geq 50 \%$ group. There were no significant differences in individual organ involvement or auto-antibodies between the two groups. Gender, education level, disease duration and SDI at recruitment, 
Fig. 1 The distribution of percentage observations in LLDAS during follow-up. A All patients; $\mathbf{B}$ patients with at least 12 months of follow-up

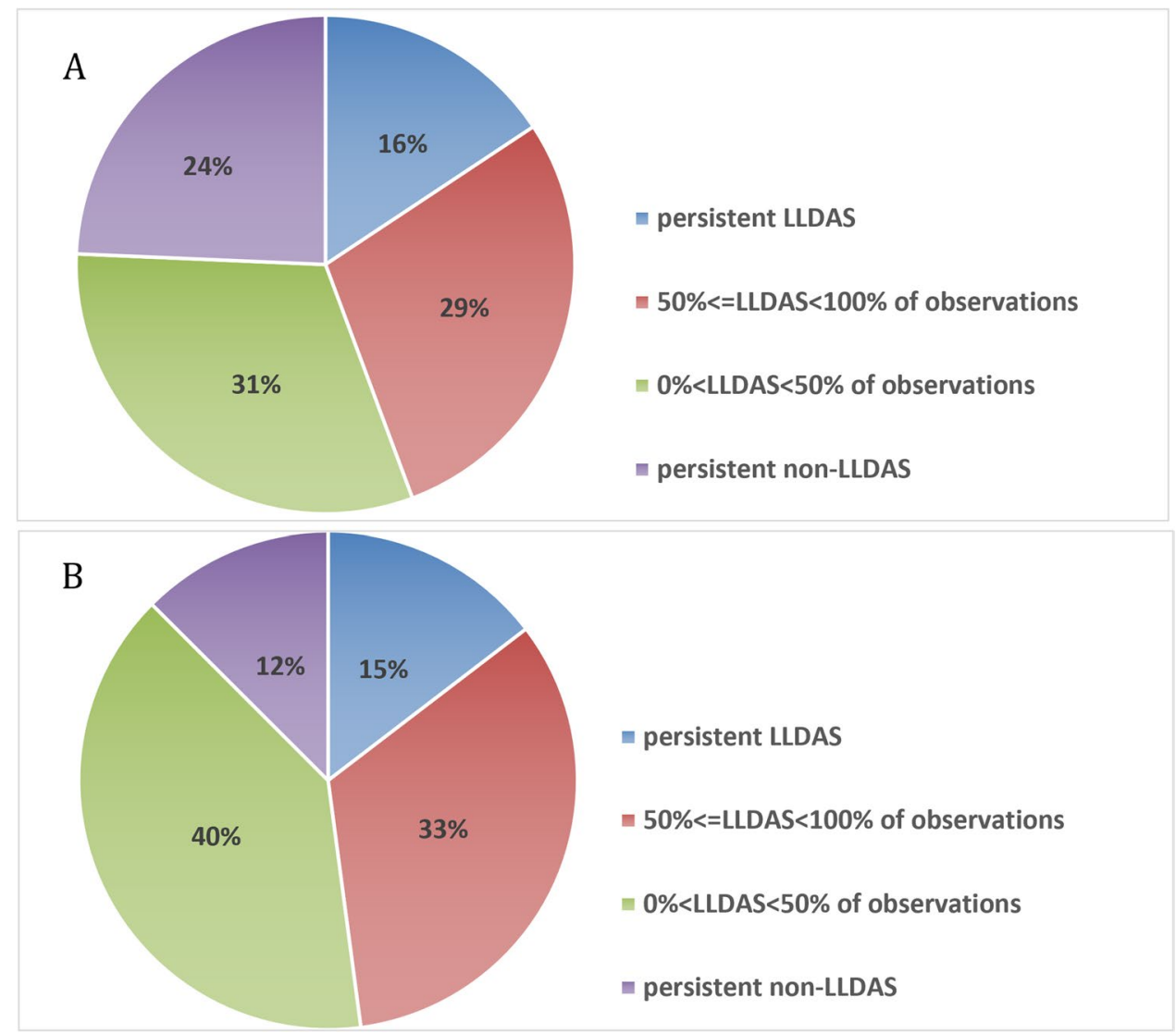

and frequency of HCQ and IS use at recruitment and during follow-up were also comparable between the two groups (Table 1).

\section{Determinants of achieving LLDAS for $\geq \mathbf{5 0} \%$ of observations}

Univariate logistic regression analyses showed that higher C3 (per $100 \mathrm{mg} / \mathrm{L})(\mathrm{OR}=1.43,95 \% \mathrm{CI}$ [1.13-1.74], $p=0.000)$ and $\mathrm{Alb}($ per $\mathrm{g} / \mathrm{L})(\mathrm{OR}=1.14,95 \% \mathrm{CI}$ [1.05-1.24], $p=0.001)$ level at recruitment were positively associated with achieving LLDAS for $\geq 50 \%$ of observations. Similarly, higher UTP (per g) $(\mathrm{OR}=0.426$, 95\%CI [0.225-0.805], $p=0.009)$ and Scr (per $10 \mu \mathrm{mol} / \mathrm{L})$ $(\mathrm{OR}=0.79,95 \% \mathrm{CI}[0.64-0.97], p=0.026)$ level at recruitment were negatively associated with achieving LLDAS for $\geq 50 \%$ of observations. In the multivariable logistic regression model, UTP (per g) $(\mathrm{OR}=0.447,95 \% \mathrm{CI}$ [0.207-0.968], $p=0.041), \mathrm{Scr}($ per $10 \mu \mathrm{mol} / \mathrm{L})(\mathrm{OR}=0.72$, $95 \% \mathrm{CI}[0.52-0.99], p=0.040)$, and C3 $(\mathrm{mg} / \mathrm{L})(\mathrm{OR}=1.60$, $95 \% \mathrm{CI}[1.18-2.17], p=0.003)$ at recruitment remained significantly associated with achieving LLDAS for $\geq 50 \%$ of observations (Table 2).

\section{Protective effect of LLDAS against flare during follow-up}

By the end of the study, 73 (39.4\%) patients had experienced a disease flare, a mild to moderate flare in 53 (28.6\%) patients and severe flare in $20(10.8 \%)$ patients. Fifty-four (23.8\%) patients flared within the first 12 months of followup and $19(10.3 \%)$ patients after 12 months.

Kaplan-Meier analyses showed that the overall cumulative flare rate was $12 \%$ at 6 months, $23 \%$ at 12 months, $45 \%$ at 24 months, and $52 \%$ at 36 months. The flare rate showed a significant difference among patients who never achieved LLDAS, LLDAS $<50 \%$, and LLDAS $\geq 50 \%$, with the lowest flare rate in the LLDAS $\geq 50 \%$ group (Log-rank $p=0.000$, Fig. 2). Meanwhile, the attainment of LLDAS achievement during the first follow-up year was associated with a reduced the risk of flare after the 1st year of follow-up (Log-rank $p=0.049$, Fig. 3).

\section{Protective effect of LLDAS on damage accrual during follow-up}

The SDI distribution at recruitment and during follow-up is shown in Supplementary Table 1. During follow-up, damage 
Table 1 Characteristics of SLE patients, categorized by proportion of follow-up observations with achievement of LLDAS

\begin{tabular}{|c|c|c|c|c|c|}
\hline Characteristics & $\begin{array}{l}\text { All patients } \\
n=185\end{array}$ & $\begin{array}{l}\text { Non-LLDAS } \\
n=45\end{array}$ & $\begin{array}{l}\text { LLDAS }<50 \%^{\Delta} \\
n=58\end{array}$ & $\begin{array}{l}\text { LLDAS } \geq 50 \%^{\Delta} \\
n=82\end{array}$ & $P$ value $^{\Delta}$ \\
\hline Female, $n(\%)$ & $163(88.1)$ & $36(80.0)$ & $50(86.2)$ & $77(93.9)$ & 0.122 \\
\hline \multicolumn{6}{|l|}{ Education level } \\
\hline Primary & $23(12.6)$ & $3(7.0)$ & $9(15.5)$ & $11(13.6)$ & \multirow[t]{3}{*}{0.946} \\
\hline Secondary & $62(34.1)$ & $14(32.6)$ & $20(34.5)$ & $28(34.6)$ & \\
\hline Tertiary & $97(53.3)$ & $26(60.5)$ & $29(50.0)$ & $42(51.9)$ & \\
\hline Age at disease onset (years) ${ }^{\dagger}$ & $33.5 \pm 14.9$ & $30.6 \pm 13.8$ & $34.3 \pm 14.5$ & $34.6 \pm 15.8$ & 0.885 \\
\hline Age at recruitment (years) & $39.0 \pm 14.9$ & $36.6 \pm 13.0$ & $39.2 \pm 15.0$ & $40.3 \pm 15.7$ & 0.670 \\
\hline Disease duration at recruitment (years) $)^{\S}$ & $2.3(0.8-7.7)$ & $1.2(0.4-8.3)$ & $2.4(0.6-7.7)$ & $2.8(1.3-6.6)$ & 0.660 \\
\hline Duration of follow-up (years) & $2.2(1.0-2.9)$ & $0.7(0.4-1.2)$ & $2.2(1.9-2.8)$ & $2.7(1.6-3.0)$ & 0.242 \\
\hline \multicolumn{6}{|l|}{ Organ involvements, $n(\%)^{\infty}$} \\
\hline Skin \& mucous involvement & $114(61.6)$ & $29(64.4)$ & $38(66.5)$ & $47(57.3)$ & 0.328 \\
\hline $\mathrm{LN}$ & $103(55.7)$ & $28(62.2)$ & $33(56.9)$ & $42(51.6)$ & 0.556 \\
\hline LN confirmed by biopsy & $62(33.5)$ & $20(44.4)$ & $19(32.8)$ & $23(28.1)$ & 0.171 \\
\hline Leukopenia & $82(44.3)$ & $20(44.4)$ & $26(44.8)$ & $36(43.9)$ & 0.914 \\
\hline Arthritis & $73(39.5)$ & $20(44.4)$ & $26(44.8)$ & $27(32.9)$ & 0.153 \\
\hline Thrombocytopenia & $47(25.4)$ & $11(21.4)$ & $14(24.1)$ & $22(26.8)$ & 0.720 \\
\hline Serositis & $28(15.1)$ & $5(11.1)$ & $10(17.5)$ & $13(16.1)$ & 0.817 \\
\hline NPSLE & $16(8.6)$ & $5(11.1)$ & $4(7.0)$ & $7(8.6)$ & 0.729 \\
\hline AIHA & $18(9.7)$ & $6(13.3)$ & $7(12.0)$ & $5(6.1)$ & 0.214 \\
\hline $\mathrm{PAH}$ & $8(4.3)$ & $3(6.7)$ & $3(5.2)$ & $2(2.4)$ & 0.391 \\
\hline \multicolumn{6}{|l|}{ Laboratories at recruitment } \\
\hline Anti-dsDNA positive, $n(\%)$ & $156(84.3)$ & $38(84.4)$ & $50(86.2)$ & $68(82.9)$ & 0.599 \\
\hline Anti-Sm positive, $n(\%)$ & $52(28.1)$ & $17(37.8)$ & $16(27.6)$ & $19(23.2)$ & 0.552 \\
\hline $\mathrm{C} 3(\mathrm{mg} / \mathrm{L})$ & $767.2 \pm 223.3$ & $667 \pm 224$ & $714 \pm 209$ & $857 \pm 200$ & $<0.001$ \\
\hline $\mathrm{C} 4(\mathrm{mg} / \mathrm{L})$ & $159.5 \pm 67.0$ & $136 \pm 73$ & $155 \pm 62$ & $174 \pm 64$ & 0.080 \\
\hline Serum albumin $(\mathrm{g} / \mathrm{L})$ & $40.4 \pm 5.7$ & $38.3 \pm 6.0$ & $39.2 \pm 5.3$ & $42.4 \pm 5.2$ & $<0.001$ \\
\hline $\mathrm{UTP}(\mathrm{g})$ & $0.15(0.04-0.95)$ & $0.67(0.17-1.78)$ & $0.39(0.05-2.3)$ & $0.05(0.02-0.15)$ & $<0.001$ \\
\hline Serum Creatinine $(\mu \mathrm{mol} / \mathrm{L})$ & $81.6 \pm 32.7$ & $86.1 \pm 48.2$ & $86.7 \pm 34.2$ & $75.6 \pm 17.2$ & 0.027 \\
\hline eGFR $\left(\mathrm{mL} / \mathrm{min} / 1.73 \mathrm{~m}^{2}\right)$ & $87.7(71.9-101.2)$ & $92.0(76.2-112.0)$ & $87.9(71.7-94.5)$ & $86.1(76.9-97.6)$ & 0.754 \\
\hline \multicolumn{6}{|l|}{ Scores at recruitment } \\
\hline SLEDAI-2 K & $2(2-6)$ & $5(2-10)$ & $4(2-6)$ & $2(0-2)$ & $<0.001$ \\
\hline PGA & $1(0-1)$ & $1(1-2)$ & $1(1-2)$ & $0(0-1)$ & $<0.001$ \\
\hline SDI & $0(0-1)$ & $0(0-1)$ & $0(0-2)$ & $0(0-1)$ & 0.311 \\
\hline \multicolumn{6}{|l|}{ Treatments } \\
\hline Prednisone daily dose $(\mathrm{mg} / \mathrm{d})$ at recruitment & $21.3 \pm 17.8$ & $34.6 \pm 17.4$ & $27.9 \pm 16.7$ & $9.1 \pm 8.8$ & $<0.001$ \\
\hline Mean prednisone daily dose $(\mathrm{mg} / \mathrm{d})$ during follow-up & $11.6 \pm 9.2$ & $22.4 \pm 11.3$ & $11.9 \pm 3.9$ & $5.4 \pm 2.6$ & $<0.001$ \\
\hline $\mathrm{HCQ}, n(\%)^{\delta}$ & $171(92.4)$ & $43(95.6)$ & $52(89.7)$ & $76(92.7)$ & 0.528 \\
\hline \multicolumn{6}{|l|}{ Immunosuppressants, $n(\%)$} \\
\hline CTX & $29(15.7)$ & $11(24.4)$ & $11(19.0)$ & $7(8.5)$ & 0.044 \\
\hline MMF & $88(47.6)$ & $20(44.4)$ & $30(51.7)$ & $38(46.3)$ & 0.731 \\
\hline CsA & $10(5.4)$ & $4(8.9)$ & $3(5.2)$ & $3(3.7)$ & 0.458 \\
\hline Tac & $6(3.2)$ & $4(8.9)$ & $0(0.0)$ & $2(2.4)$ & 0.035 \\
\hline AZA & $20(10.8)$ & $5(11.1)$ & $4(6.9)$ & $11(13.4)$ & 0.472 \\
\hline MTX & $16(8.7)$ & $2(4.4)$ & $7(12.1)$ & $7(8.6)$ & 0.395 \\
\hline LEF & $21(11.4)$ & $10(22.2)$ & $3(5.2)$ & $8(9.8)$ & 0.021 \\
\hline Flare during follow-up & $73(40.1) / 182$ & $17(38.6) / 44$ & $34(58.6) / 58$ & $22(27.5) / 80$ & $<0.001$ \\
\hline Flare times during follow-up & $0(0-1)$ & $0(0-1)$ & $1(0-1)$ & $0(0-1)$ & $<0.001$ \\
\hline Damage accrual of year 1 & $21(14.9) / 141$ & $3(15) / 20$ & $11(20) / 55$ & $7(10.6) / 66$ & 0.150 \\
\hline
\end{tabular}


Table 1 (continued)

\begin{tabular}{|c|c|c|c|c|c|}
\hline Characteristics & $\begin{array}{l}\text { All patients } \\
n=185\end{array}$ & $\begin{array}{l}\text { Non-LLDAS } \\
n=45\end{array}$ & $\begin{array}{l}\operatorname{LLDAS}<50 \%^{\Delta} \\
n=58\end{array}$ & $\begin{array}{l}\operatorname{LLDAS} \geq 50 \%^{\Delta} \\
n=82\end{array}$ & $P$ value $^{\Delta}$ \\
\hline Damage accrual of year 2 & $37(35.6) / 104$ & $4(80) / 5$ & $21(48.8) / 43$ & $12(21.4) / 56$ & 0.004 \\
\hline
\end{tabular}

Data are presented as mean \pm standard deviation for normally distributed continuous variables, median (IQR) for abnormally distributed continuous variables and numbers (percentages) for categorical variables

$\Delta$ non-LLDAS group was defined as patients who had never achieved LLDAS during follow-up, LLDAS $<50 \%$ group was defined as patients who achieved LLDAS less than 50\% of follow-up time, and LLDAS $\geq 50 \%$ group was defined as patients who achieved LLDAS at least $50 \%$ of follow-up time. The comparison was made between LLDAS $<50 \%$ and LLDAS $\geq 50 \%$ group

${ }^{\dagger}$ Disease onset defined as the date of first symptom related to SLE

${ }^{\ddagger}$ Recruitment defined as the first date of being recruited in the cohort

$\S^{\S}$ Disease duration at recruitment defined as time from disease onset to recruitment

${ }^{\mathbb{I}}$ Duration from recruitment to last visit

${ }^{\infty}$ Present ever during course of disease

${ }^{\delta} \mathrm{Hydroxychloroquine,} \mathrm{One} \mathrm{IS} \mathrm{or} \mathrm{the} \mathrm{combination} \mathrm{of} \mathrm{two} \mathrm{ISs} \mathrm{were} \mathrm{used} \mathrm{for} \mathrm{at} \mathrm{least} 3$ months during follow-up

Abbreviations: LN lupus nephritis; AIHA autoimmune hemolytic anemia; NPSLE neuropsychiatric SLE; PAH pulmonary arterial hypertension; $A N A$ anti-nuclear antibody; anti-dsDNA anti-double-stranded DNA antibody; $C 3$ complement 3 ; $C 4$ complement 4; UTP 24-h urine total protein; $e G F R$ estimated glomerular filtration rate; SLEDAI SLE disease activity index; PGA patient global assessment; SDI Systemic Lupus International Collaborating Clinics damage index; $H C Q$ hydroxychloroquine; $C T X$ : cyclophosphamide; $M M F$ mycophenolate mofetil; $C s A$ cyclosporin; Tac tacrolimus; AZA azathioprine; MTX methotrexate; $L E F$ leflunomide

accrual was observed in $21 / 141$ (14.9\%) patients within 1 year, 37/104 (35.6\%) patients within 2 years, and 11/21 (52.4\%) patients within 3 years. In a multivariable logistic regression model, age at disease onset and duration of follow-up (per year) were independent risk factors for damage accrual ( $\mathrm{OR}=1.05,95 \% \mathrm{CI}[1.01-1.11], p=0.047$; and $\mathrm{OR}=6.94,95 \% \mathrm{CI}[1.89-25.44], p=0.003$; respectively), and achieving LLDAS at least $50 \%$ of observations during follow-up had a significant protective effect on damage accrual (OR $=0.19,95 \%$ CI [0.04-0.99], $p=0.049$ ) (Supplementary Table 2).

\section{Discussion}

LLDAS, an outcome measure developed by the multinational Asia-Pacific Lupus Collaboration, has been validated retrospectively and prospectively to be associated with a reduced risk of organ damage in studies from Latin America, North America, Europe, and the Asia-Pacific region [6, 9, 24]. Here, we present a prospective single-center longitudinal study of LLDAS attainment and its association with disease flare and damage accrual in Chinese patients.

In our cohort, $75.1 \%$ of the patients achieved LLDAS at least once and $44.3 \%$ were in LLDAS at least $50 \%$ of visits, which were comparable with other reports [7, 25] and confirmed that LLDAS is an achievable goal, even in newly diagnosed patients who are more likely to have active disease [26].

Our analyses showed that there were significant differences in baseline characteristics of patients who achieved
LLDAS for at least $50 \%$ of follow-up observations and those who did not, in terms of baseline UTP, Scr, Alb, and C3. As expected, the variables which are directly involved in the LLDAS assessment criteria such as SLEDAI-2 K, PGA, and prednisone dose were also significantly different between the two groups. Compared with other serological markers such as $\mathrm{C} 4$ and anti-dsDNA, baseline $\mathrm{C} 3$ was shown in logistic regression analyses to be the most significant marker for subsequent LLDAS attainment. Two previous studies showed that renal involvement was negatively associated with achieving LLDAS $[7,27]$, but the present study identified that two more specific indicators (UTP and Scr) of renal involvement were independent negative predictive factors for achieving LLDAS $\geq 50 \%$ of observations. Of course, we need to clarify that the patients with $\mathrm{LN}$ accounted for more than $50 \%$ of all patients in this cohort. In case of without these $\mathrm{LN}$ patients, the predicting effect of UTP and Scr would not be statistically significant. A series of epidemiological studies had consistent results with us that nephropathy is one of the most common major organ involvements in SLE patients in China and Asian-Pacific region $[1,7,28]$. The present study further emphasizes the critical role of renal involvement in affecting therapeutic target achievement.

The present study revealed that percentage of time in LLDAS was negatively associated with disease flare. Disease flare is often managed with the use of glucocorticoids and IS, which in turn may lead to further damage accrual or infection risk. A study from the Netherlands showed that having at least one major flare was associated with future damage accrual [25]. The recommendations from 
Table 2 Determinants of achieving LLDAS in at least $50 \%$ of observations by univariate and multivariable logistic regression analysis

\begin{tabular}{|c|c|c|c|c|c|c|}
\hline \multirow[t]{2}{*}{ Variables } & \multicolumn{3}{|c|}{ Univariate analysis } & \multicolumn{3}{|c|}{ Multivariable analysis } \\
\hline & OR & $95 \% \mathrm{CI}$ & $P$ value & OR & $95 \% \mathrm{CI}$ & $P$ value \\
\hline Gender: female & 2.46 & $0.76-7.96$ & 0.132 & & & \\
\hline Education level: tertiary & 1.08 & $0.67-.71$ & 0.760 & & & \\
\hline Age at disease onset (per year) ${ }^{\dagger}$ & 1.00 & $0.98-1.02$ & 0.886 & & & \\
\hline Disease duration at recruitment (per year) ${ }^{\ddagger}$ & 1.011 & $0.96-1.07$ & 0.658 & & & \\
\hline Follow-up duration (per year) $)^{\S}$ & 0.95 & $0.63-1.45$ & 0.821 & & & \\
\hline \multicolumn{7}{|l|}{ Organ involvements ${ }^{\mathrm{I}}$} \\
\hline Skin \& mucous involvement & 0.71 & $0.35-1.42$ & 0.329 & & & \\
\hline Arthritis & 0.75 & $0.37-1.50$ & 0.414 & & & \\
\hline Serositis & 1.19 & $0.27-5.19$ & 0.816 & & & \\
\hline $\mathrm{LN}$ & 0.97 & $0.50-1.91$ & 0.934 & & & \\
\hline LN confirmed by biopsy & 0.54 & $0.27-1.07$ & 0.076 & & & \\
\hline NPSLE & 1.44 & $0.25-8.11$ & 0.682 & & & \\
\hline AIHA & 0.44 & $0.12-1.65$ & 0.226 & & & \\
\hline Thrombocytopenia & 1.47 & $0.65-3.35$ & 0.358 & & & \\
\hline Leukopenia & 0.79 & $0.38-1.62$ & 0.513 & & & \\
\hline PAH & 0.46 & $0.07-2.83$ & 0.401 & & & \\
\hline \multicolumn{7}{|l|}{ Laboratories at recruitment } \\
\hline Anti-dsDNA positive & 0.82 & $0.41-1.62$ & 0.564 & & & \\
\hline Anti-Sm positive & 0.79 & $0.37-1.71$ & 0.553 & & & \\
\hline C3 (per 100 mg/L) & 1.43 & $1.18-1.74$ & 0.000 & 1.60 & $1.18-2.17$ & 0.003 \\
\hline C4 (per 100 mg/L) & 1.64 & $0.94-2.88$ & 0.084 & & & \\
\hline Serum albumin (per g/L) & 1.14 & $1.05-1.24$ & 0.001 & 1.07 & $0.94-1.22$ & 0.283 \\
\hline UTP (per g) & 0.426 & $0.225-0.805$ & 0.009 & 0.447 & $0.207-0.968$ & 0.041 \\
\hline Serum creatinine (per $10 \mu \mathrm{mol} / \mathrm{L}$ ) & 0.79 & $0.64-0.97$ & 0.026 & 0.72 & $0.52-0.99$ & 0.040 \\
\hline eGFR $\left(\right.$ per $\left.\mathrm{mL} / \mathrm{min} / 1.73^{2}\right)$ & 0.99 & $0.98-1.01$ & 0.417 & & & \\
\hline \multicolumn{7}{|l|}{ Scores at recruitment } \\
\hline SDI & 0.80 & $0.62-1.03$ & 0.079 & & & \\
\hline \multicolumn{7}{|l|}{ Treatments $^{\delta}$} \\
\hline $\mathrm{HCQ}^{\delta}$ & 1.46 & $0.45-4.78$ & 0.530 & & & \\
\hline $\mathrm{CTX}^{\delta}$ & 0.40 & $0.14-1.10$ & 0.076 & & & \\
\hline $\mathrm{MMF}^{\delta}$ & 0.81 & $0.41-1.58$ & 0.530 & & & \\
\hline $\mathrm{CsA}^{\delta}$ & 0.70 & $0.14-3.58$ & 0.665 & & & \\
\hline $\mathrm{AZA}^{\delta}$ & 2.09 & $0.63-2.93$ & 0.227 & & & \\
\hline MTX $^{\delta}$ & 0.69 & $0.23-2.08$ & 0.510 & & & \\
\hline $\mathrm{LEF}^{\delta}$ & 1.98 & $0.50-7.82$ & 0.328 & & & \\
\hline
\end{tabular}

†Years; disease onset defined as the date of first symptom related to SLE

${ }^{\ddagger}$ Years; disease duration at recruitment defined as time from disease onset to recruitment

${ }^{\S}$ Duration from recruitment to last visit

"Present ever during course of disease

${ }^{8}$ Hydroxychloroquine or IS was used for at least 3 months during follow-up

Abbreviations: LN lupus nephritis; NPSLE neuropsychiatric SLE; AIHA autoimmune hemolytic anemia; $P A H$ pulmonary arterial hypertension; anti-dsDNA anti-double-stranded DNA antibody; $C 3$ complement 3; UTP 24-h urine total protein; $e G F R$ estimated glomerular filtration rate; SDI Systemic Lupus International Collaborating Clinics damage index; $H C Q$ hydroxychloroquine; $C T X$ cyclophosphamide; $M M F$ mycophenolate mofetil; $C s A$ cyclosporin; $A Z A$ azathioprine; $M T X$ methotrexate; $L E F$ leflunomide the SLE-T2T taskforce underlined that prevention of flares should be a therapeutic goal in SLE and the present study identifies LLDAS as an achievable and important target for preventing flare. These data are encouraging for the future application of the LLDAS in clinical practice as a treatment target. 
Fig. 2 Occurrence of flare curves during follow-up (Kaplan-Meier analysis). The comparison of flare rate was among patients who achieved LLDAS for more than $50 \%$ of observations, those who achieved LLDAS less than $50 \%$ of observations, and those who never achieved LLDAS (log rank $p=0.000$ )

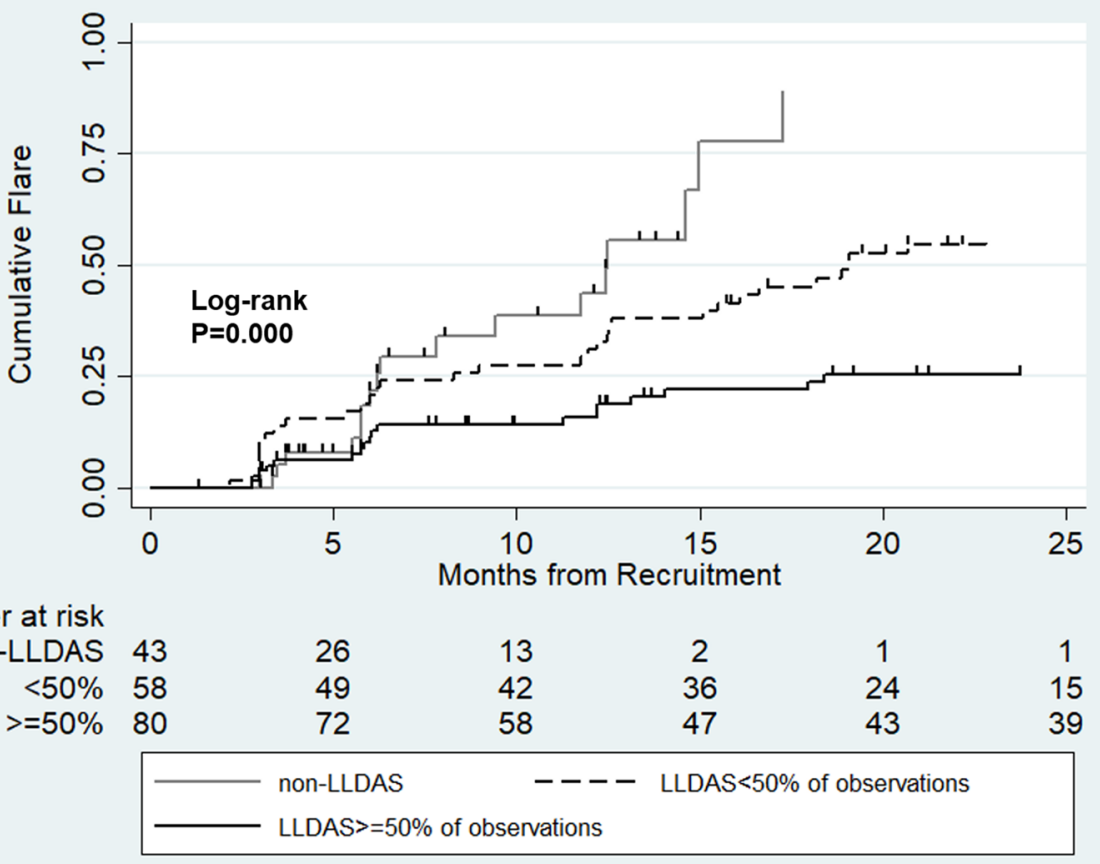

Fig. 3 Flare in the second year of follow-up (Kaplan-Meier analysis) in patients categorized by time spent in LLDAS (LLDAS more than $50 \%$ of observations, LLDAS less than $50 \%$ of observations, and never achieved LLDAS) during the first year of follow-up (log rank $p=0.049$ )

In the present cohort, we found $14.9 \%$ of patients had damage accrual within 1 year, and we observed a significant protective association of LLDAS with damage accrual. Achieving LLDAS $\geq 50 \%$ of observations was an independent protective factor for damage accrual. Early damage accrual is a particularly important outcome indicator for SLE patients as it is predictive of further damage accrual
[29] and lower survival. For example, one study showed that patients with initial damage had a fourfold higher mortality rate compared to those with no early damage [30]. Another study showed that damage 1 year after diagnosis was a significant predictor of death within 10 years of diagnosis [31].

Interestingly, age of disease onset was found to have different associations with disease flare and damage accrual, 
wherein older age at disease onset was an independent protective factor for disease flare, while it was an independent risk factor for damage accrual. One study found that patients with younger age at disease onset were more likely to have active disease than those with older age at disease onset [32]. This implies that more vigilant care is needed for these patients. However, consistent with another report [8], older patients were more likely to develop permanent organ damage which need closer monitor and prophylactic treatment.

There are some limitations to this study. First, our sample size was relatively small, the follow-up duration was relatively short especially in those who did not achieve LLDAS, and the loss to follow-up ratio was relatively high, which limits the evaluation for organ damage development and the correlation between LLDAS achievement and damage accrual. Secondly, our assumption that patients are in LLDAS for the entire duration between two visits at which they are found to be in LLDAS may overestimate the duration of being in LLDAS.

In conclusion, LLDAS was an attainable goal in clinical practice in Chinese SLE patients and therefore a potentially applicable goal in a T2T strategy. Higher urine protein and serum creatinine level and lower $\mathrm{C} 3$ level at baseline were negative influence factors for achieving LLDAS subsequently. LLDAS is a promising treatment target in SLE, negatively associated with disease flare and damage. Future prospective studies, using large sample sizes, are expected to validate these findings.

Supplementary Information The online version contains supplementary material available at https://doi.org/10.1007/s10067-021-05940-z.

Acknowledgements The data in this cohort was collected and recorded using the framework of the LLDAS study initiated by the APLC.

Author contribution All authors were involved in drafting the manuscript or revising it critically for important intellectual content, and all authors approved the final version to be published. YH had full access to all the data in the study and made substantial contributions to study design, data collection, data analysis, interpretation of results, and preparation of manuscript. SO made substantial contributions to preparation of the manuscript. LJ, DG, YF, YG, XZ, and GL made substantial contributions to data collection and manuscript revision. EM and MN made substantial contributions to study design and preparation of the manuscript. ZZ had full access to all the data in the study and takes responsibility for the integrity of the data and the accuracy of the data analysis.

Data availability The datasets analyzed during the current study are available from the corresponding author upon reasonable request.

\section{Declarations}

Ethics approval and consent to participate Ethics approval for data collection was obtained from the ethics committee of Peking University First Hospital (Project Number: 2017[1284]), and written informed consent was obtained from all participants prior to study enrolment.
Disclosures None.

Open Access This article is licensed under a Creative Commons Attribution 4.0 International License, which permits use, sharing, adaptation, distribution and reproduction in any medium or format, as long as you give appropriate credit to the original author(s) and the source, provide a link to the Creative Commons licence, and indicate if changes were made. The images or other third party material in this article are included in the article's Creative Commons licence, unless indicated otherwise in a credit line to the material. If material is not included in the article's Creative Commons licence and your intended use is not permitted by statutory regulation or exceeds the permitted use, you will need to obtain permission directly from the copyright holder. To view a copy of this licence, visit http://creativecommons.org/licenses/by/4.0/.

\section{References}

1. Lin Mu, Hao Y, Hong Huang $X$, Yang AX, Zhang $X$, Ji L, Geng Y, Zhang Z (2018) Mortality and prognostic factors in Chinese patients with systemic lupus erythematosus. Lupus 27(10): 1742-1752

2. Lee YH, Choi SJ, Ji JD, Song GG (2016) Overall and causespecific mortality in systemic lupus erythematosus: an updated meta-analysis. Lupus 25(7):727-734

3. Franklyn K, Hoi A, Nikpour M, Morand FM (2014) The need to define treatment goals for systemic lupus erythematosus. Nat Rev Rheumatol 10(9):567-571

4. Van Vollenhoven R F, MoscaM, BertsiasG, Isenberg D, Kuhn A, Lerstrøm K, et al (2014) Treat-to-target in systemic lupus erythematosus: recommendations from an international task force. Ann Rheum Dis 73(6): 958-967

5. van Vollenhoven R, Voskuyl A, Bertsias G et al (2017) A framework for remission in SLE: consensus findings from a large international task force on definitions of remission in SLE (DORIS). Ann Rheum Dis 76:554-561

6. Golder V, Kandane-Rathnayake R, Huq M, Nim HT, Louthrenoo W, Luo SF et al (2019) Lupus low disease activity state as a treatment endpoint for systemic lupus erythematosus: a prospective validation study. The Lancet Rheumatology 1(2):e95-e102

7. Franklyn K, Lau CS, Navarra SV, Louthrenoo W, Lateef A, Hamijoyo L et al (2016) Definition and initial validation of a Lupus Low Disease Activity State (LLDAS). Ann Rheum Dis 75(9):1615-1621

8. Zen M, Iaccarino L, Gatto M, Saccon F, Larosa M, Ghirardello A, Punzi L, Doria A (2018) Lupus low disease activity state is associated with a decrease in damage progression in Caucasian patients with SLE, but overlaps with remission. Ann Rheum Dis 77(1):104-110

9. Sharma C, Raymond W, Eilertsen G, Nossent J (2020) Association of achieving lupus low disease activity state fifty percent of the time with both reduced damage accrual and mortality in patients with systemic lupus erythematosus. Arthritis Care Res (Hoboken) 72(3):447-451

10. Vera Golder, Rangi Kandane-Rathnayake, Molla Huq, Worawit Louthrenoo,Shue FenLuo, Yeong-Jian Jan Wu, et al. Evaluation of remission definitions for systemic lupus erythematosus: a prospective cohort study. The Lancet Rheumatology 1(2): e103-e110.

11. Hochberg MC (1997) Updating the American College of Rheumatology revised criteria for the classification of systemic lupus erythematosus. Arthritis Rheum 40(9):1725

12. Petri M, Orbai AM, Alarcon GS, Gordon C, Merrill JT, Fortin PR et al (2012) Derivation and validation of the Systemic Lupus 
International Collaborating Clinics classification criteria for systemic lupus erythematosus. Arthritis Rheum 64(8):2677-2686

13. Ibanez D, Gladman DD, Urowitz MB (2005) Adjusted mean Systemic Lupus Erythematosus Disease Activity Index-2K is a predictor of outcome in SLE. J Rheumatol 32(5):824-827

14. Petri M, Hellmann D, Hochberg M (1992) Validity and reliability of lupus activity measures in the routine clinic setting. J Rheumatol 19(1):53-59

15. Petri M, Buyon J, Kim M (1999) Classification and definition of major flares in SLE clinical trials. Lupus 8(8):685-691

16. Gladman DD, Goldsmith $\mathrm{CH}$, Urowitz MB, Bacon P, Fortin P, Ginzler E et al (2000) The Systemic Lupus International Collaborating Clinics/American College of Rheumatology (SLICC/ACR) Damage Index for Systemic Lupus Erythematosus International Comparison. J Rheumatol 27(2):373-376

17. Ware JE Jr, Sherbourne CD. The MOS 36-item short-form health survey (SF-36). I. Conceptual framework and item selection (1992). Med Care. 30(6): 473-483.

18. Kandane-Rathnayake R, Golder V, Louthrenoo W, Luo SF, Jan Wu YJ, Li Z et al (2019) Development of the Asia Pacific Lupus Collaboration cohort. Int J Rheum Dis 22(3):425-433

19. Tan EM, Cohen AS, Fries JF, Masi AT, McShane DJ, Rothfield NF, Schaller JG, Talal N, Winchester RJ (1982) The 1982 revised criteria for the classification of systemic lupus erythematosus. Arthritis Rheum 25(11):1271-1277

20. [No authors listed], (1999) The American College of Rheumatology nomenclature and case definitions for neuropsychiatric lupus syndromes. Arthritis Rheum 42(4):599-608

21. Hermosillo-Romo D, Brey RL (2002) Diagnosis and management of patients with neuropsychiatric systemic lupus erythematosus (NP-SLE). Best Pract Res Clin Rheumatol 16(2):229-244

22. Gehrs BC, Friedberg RC (2002) Autoimmune hemolytic anemia. Am J Hematol 69(4):258-271

23. Galiè N, Humbert M, Vachiery JL, Gibbs S, Lang I, Torbicki A et al (2016) 2015ESC/ERS Guidelines for the diagnosis and treatment of pulmonary hypertension: the joint task force for the diagnosis and treatment of pulmonary hypertension of the European Society of Cardiology (ESC) and the European Respiratory Society (ERS): Endorsed by: Association for European Paediatric and Congenital Cardiology (AEPC), International Society for Heart and Lung Transplantation (ISHLT). Eur Heart J 37(1):67-119

24. Ugarte-Gil MF, Wojdyla D, Pons-Estel GJ, Catoggio LJ, Drenkard C, Sarano J et al (2017) Remission and Low Disease Activity
Status (LDAS) protect lupus patients from damage occurrence: data from a multiethnic, multinational Latin American Lupus Cohort (GLADEL). Ann Rheum Dis 76(12):2071-2074

25. Tsang-A-Sjoe MWP, P M, Bultink M I, Heslinga M, Voskuyl E A, (2017) Both prolonged remission and Lupus Low Disease Activity State are associated with reduced damage accrual in systemic lupus erythematosus. Rheumatology 56(1):121-128

26. Urowitz MB, Gladman DD, Ibanez D, Fortin PR, Bae SC, Gordon $C$ et al (2012) Evolution of disease burden over five years in a multicenter inception systemic lupus erythematosus cohort. Arthritis Care Res (Hoboken) 64(1):132-137

27. Vera G, Rangi K-R, Alberta Yik-Bun H, Huq M, Louthrenoo W, An $\mathrm{Y}$ et al (2016) Frequency and predictors of the lupus low disease activity state in a multi-national and multi-ethnic cohort. Arthritis Res Ther 18(1):260-268

28. Li M, Zhang W, Leng X, Li Z, Ye Z, Li C, et al. (2013) Chinese SLE Treatment and Research group (CSTAR) registry: I. Major clinical characteristics of Chinese patients with systemic lupus erythematosus. Lupus. 22(11):1192-1199

29. Alarcon GS, Roseman JM, McGwin G Jr, Uribe A, Bastian M H, Fessler J B, Baethge A B, Friedman W A, Reveille D J, LUMINA Study Group (2004) Systemic lupus erythematosus in three ethnic groups. XX. Damage as a predictor of further damage. Rheumatology 43(2): 202-205

30. Rahman P, Gladman D, Urowitz MB, Ahmad B, Shah SM, Tahir S, Merchant D, Ahmad A (2001) Early damage as measured by the SLICC/ACR damage index is a predictor of mortality in systemic lupus erythematosus. Lupus 10(2):93-96

31. Rabbani MA, Habib HB, Islam M, Ahmad B, Shah AMS, Tahir S, Merchant D, Ahmad A (2010) Early renal damage assessed by the SLICC/ACR damage index is predictor of severe outcome in lupus patients in Pakistan. Lupus 19(13):1573-1578

32. Aljohani R, Gladman DD, Su J, Urowitz MB (2017) Disease evolution in late-onset and early-onset systemic lupus erythematosus. Lupus 26(11):1190-1196

Publisher's note Springer Nature remains neutral with regard to jurisdictional claims in published maps and institutional affiliations.

\section{Authors and Affiliations}

\section{Yanjie Hao ${ }^{1,2} \oplus$. Shereen Oon ${ }^{2,3} \cdot{\text { Lanlan } \mathrm{Ji}^{1} \cdot \text { Dai Gao }}^{1}$ - Yong Fan ${ }^{1} \cdot$ Yan Geng $\cdot$ Xiaohui Zhang ${ }^{1} \cdot$ Guangtao $\mathrm{Li}^{1}$. Eric F. Morand ${ }^{4} \cdot$ Mandana Nikpour $^{2,3} \cdot$ ZhuoLi Zhang $^{1}$}

1 Department of Rheumatology and Clinical Immunology, Peking University First Hospital, 8 Xishiku St, Beijing 100034, People's Republic of China

2 The University of Melbourne Department of Medicine at St. Vincent's Hospital Melbourne, 41 Victoria Parade, Fitzroy, VIC 3065, Australia
3 Department of Rheumatology, St. Vincent's Hospital Melbourne, 41 Victoria Parade, Fitzroy, VIC 3065, Australia

4 Monash University, Faculty of Medicine, Nursing and Health Sciences, Melbourne, Australia 